\title{
Joint and Distributed Linear Precoding for Centralised and Decentralised Multicell Processing
}

\author{
Rong Zhang and Lajos Hanzo \\ School of ECS., Univ. of Southampton, SO17 1BJ, UK. \\ Tel: +44-23-80-593 125, Fax: +44-23-80-593 045 \\ Email: rz,lh@ecs.soton.ac.uk, http://www-mobile.ecs.soton.ac.uk
}

\begin{abstract}
Linear precoding techniques are designed for both joint and distributed Multi-Cell Processing (MCP), where both centralised and decentralised Channel State Information (CSI) exchange scenarios are considered. The outage rate of various linear precoding techniques is investigated in the cell-edge area of a practical three-cell based cooperative scenario under both fullyloaded and lightly-loaded system configurations, when encountering either no tier-one Co-Channel Interference (CCI) or full tier-one CCI. The numerical results demonstrate the superiority of the low-complexity, yet scalable decentralised Distributed Signal-to-Leakage-Noise-Ratio (DSLNR) based linear precoding technique under realistic CSI quantisation and channel-induced feedback error conditions when full tier-one CCI is considered.
\end{abstract}

\section{INTRODUCTION}

Multiple Input Multiple Output (MIMO) system based Multi-Cell Pre-processing (MCP) [1], [2] constitutes a promising enabler for improving the throughput of cell-edge Mobile Stations (MS). A MCP based cooperative transmission regime has to share the data of all the Base Stations (BSs) involved for jointly processing them. This is typically achieved by assuming the existence of a Central Unit (CU), which connects all the BSs considered via a reliable high-speed optical fibre.

Moreover, MCP requires Channel State Information at all the Distributed Transmitters (CSI-DT). There are two different MCP frameworks for sharing the CSI-DT, namely the centralised and decentralised framework [3]. More explicitly, the centralised framework has to exchange the CSI of all the BSs involved with the aid of the CU, while the decentralised framework gathers the CSI of all the BSs involved at each BS locally. In practice, the CSI-DT may suffer from both quantisation noise as well as feedback errors [4]. This undesirable phenomenon dominates the achievable MCP performance, when various Down-Link (DL) linear precoding techniques are employed. In the context of MCP, linear BS precoding techniques may be implemented in either a joint or distributed fashion. Linear joint DL precoding techniques determine the precoding matrix for all the BSs involved globally. By contrast, distributed linear precoding techniques optimise the DL precoding matrix of each individual BS locally.

Although individual reports on the attainable MCP performance of linear precoding techniques may be found in the literature, they are based on different system configurations

Acknowledgements: The financial support of the EPSRC under the auspices of the UK-India Advanced Technology Centre in Wireless Communications and that of the EU's OPTIMIX project is gratefully acknowledged. associated with different assumptions. This motivates us to provide a comparative study of the various joint and distributed linear precoding techniques for both centralised and decentralised CSI-DT scenarios in the presence of potential CSI feedback errors.

The rest of the paper is organised as follows. In Section II, we discuss various linear precoding techniques and introduce both the centralised and decentralised MCP framework relying on quantised CSI. In Section III, the attainable performance of various linear precoding techniques recorded for both fully-loaded and lightly-loaded scenarios is investigated and compared. Finally, we conclude our discourse in Section IV.

Notation: Throughout the paper, lower (upper) case boldface letters represent column vectors (matrices). The matrix $\mathbf{I}_{a}$ represents $(a \times a)$ identity matrix, while $\mathbf{I}$ represents $\left(N_{r} \times N_{r}\right)$ identity matrix by default, where $N_{r}$ denotes the number of received antennas at each MS. The superscript $(\cdot)^{T}$ denotes transposition and $(\cdot)^{H}$ represents the conjugate transpose.

\section{SYSTEM DESCRIPTION OF MCP}

\section{A. Cellular Topology and System Model}

Let us first introduce the cellular topology of Fig 1, where the hexagonal three-sector cellular model associated with a unity frequency reuse is employed. Let $\mathcal{B}=\left\{\mathcal{B}_{c}, \mathcal{B}_{o}\right\}$ denote the set containing all BSs considered, where $\mathcal{B}_{c}$ hosts the BSs involved in the cooperative transmission and $\mathcal{B}_{O}$ hosts the rest of the BSs. Although in principle $\left|\mathcal{B}_{c}\right|=|\mathcal{B}|$ is possible, where $|\cdot|$ denotes the cardinality of a set, in order to reduce the complexity of BS cooperation, $\left|\mathcal{B}_{c}\right|=3$ represents a realistic scenario, where three adjacent BSs form a joint cooperative transmission site, as indicated by the dashed area in Fig 1. Hence the cell-edge of the conventional cells effectively becomes the cell-centre of the newly formed cooperative site, as indicated by the shadowed circle in Fig 1. These geographically separated BSs are connected to a common $\mathrm{CU}$ via a high-rate lossless optical fibre, where the CU is responsible for performing high-level decisions, such as transmission scheduling, resource management and precoding matrix allocation. Since in practice we have $\left|\mathcal{B}_{c}\right|<\mathcal{B}$, the residual CCI imposed by the active transmissions in the noncooperating $\mathrm{BS}$ set $\mathcal{B}_{o}$ may remain considerable. Hence, we take into account the most dominant tier-one CCI, as seen in Fig 1 , where $\left|\mathcal{B}_{o}\right|=9$ is observed.

A general cooperative scenario is constituted by $\left|\mathcal{B}_{c}\right|=N_{b}$ BSs, where each BS is equipped with $N_{t}$ transmit antennas. 


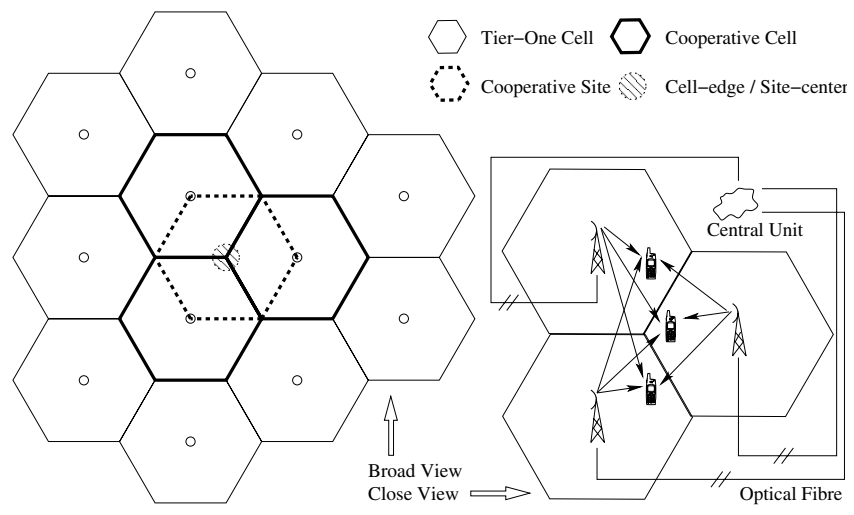

Fig. 1. The cellular topology considered and multicell processing

Let us assume that a total of $N_{u}=N_{b}$ MSs each equipped with $N_{r}$ receive antennas are involved in the cooperative scenario, where each of the $N_{u}$ MSs roams within the coverage area of different BS, which is often referred to as its anchor BS or home BS as portrayed in Fig 1. Furthermore, we assume that each MS has $N_{s}$ multiplexed data streams. Hence, the cooperative scenario may be described by the parameter combination $\left\{N_{b}, N_{t}, N_{u}, N_{r}, N_{s}\right\}$, in addition, we let $N_{T}=$ $\left(N_{b} \times N_{t}\right)$ and $N_{R}=\left(N_{u} \times N_{r}\right)$ denote the total number of transmitter and receiver antennas, respectively.

1) SCP: In a SCP scenario, each MS is only served by its anchor BS, while the remaining active transmissions of all other BSs are considered as CCI. Hence, the discrete-time model of the received signal at MS $j$ may be written as:

$$
\mathbf{y}_{j}=\mathbf{H}_{j, j} \mathbf{G}_{j, j} \mathbf{x}_{j}+\sum_{i \in \mathcal{B}_{c,-j}} \mathbf{H}_{i, j} \mathbf{G}_{i, i} \mathbf{x}_{i}+\mathbf{\Psi}+\mathbf{n}_{j},
$$

where the first three terms of Eq (1) represent the desired signal of MS $j$, the CCI imposed by the BSs of the cooperative site excluding the $j$ th BS hosted in the set $\mathcal{B}_{c,-j}$ and the CCI imposed by all tier-one BSs hosted in the set $\mathcal{B}_{o}$, respectively, where $\mathbf{\Psi}=\sum_{i \in \mathcal{B}_{o}} \mathbf{H}_{i, j} \mathbf{G}_{i, i} \mathbf{x}_{i}$.

Additionally, $\mathbf{y}_{j} \in \mathbb{C}^{N_{r}}$ and $\mathbf{n}_{j} \in \mathbb{C}^{N_{r}}$ denote the received signal vector and the circularly symmetric complex Gaussian noise having a covariance matrix of $\mathbf{R}_{n}=N_{0} \mathbf{I}$, respectively. Furthermore, $\mathbf{H}_{i, j} \in \mathbb{C}^{N_{r} \times N_{t}}, i \in \mathcal{B}$ denotes the MIMO channel between the $i$ th BS and the $j$ th MS, which includes the pathloss, shadowing and fast-fading components. $\mathbf{G}_{i, i} \in$ $\mathbb{C}^{N_{t} \times N_{s}}, i \in \mathcal{B}$ denotes the linear precoding matrix employed at the $i$ th BS. Finally, $\mathbf{x}_{i} \in \mathbb{C}^{N_{s}}, i \in \mathcal{B}$ denotes the transmitted data streams that have i.i.d. zero-mean unit-variance complex Gaussian entries, which are assumed to be independent of both the noise and of the MIMO channel, obeying $\mathbb{E}\left(\mathbf{x}_{i} \mathbf{x}_{i}^{H}\right)=\mathbf{I}_{N_{s}}$.

In order to maintain the per-BS power constraint $P_{B S}$ that is the same for all BSs, we further let $\operatorname{Tr}\left[\mathbf{G}_{i, i} \mathbf{G}_{i, i}^{H}\right]=P_{B S}$. In this paper, we consider an equal power allocation in the SCP scenario, when a single-user detector is employed as a benchmarker, which is unaware of the CCI, the achievable rate of the SCP scheme is given as:

$\mathcal{R}_{s}=\frac{1}{N_{u}} \sum_{j=1}^{N_{u}} \log \left|\mathbf{I}+\frac{\mathbf{H}_{j, j} \mathbf{G}_{j, j} \mathbf{G}_{j, j}^{H} \mathbf{H}_{j, j}^{H}}{\mathbf{R}_{I}+\sum_{i \in \mathcal{B}_{c,-j}} \mathbf{H}_{i, j} \mathbf{G}_{i, i} \mathbf{G}_{i, i}^{H} \mathbf{H}_{i, i}^{H}}\right|$, where $\mathbf{R}_{I}=N_{0} \mathbf{I}+\sum_{i \in \mathcal{B}_{o}} \mathbf{H}_{i, j} \mathbf{G}_{i, i} \mathbf{G}_{i, i}^{H} \mathbf{H}_{i, j}^{H}$ denotes the covariance of the tier-one CCI plus noise.

2) MCP: In a MCP scenario, each MS is jointly served by all BSs in the cooperative site, where the only CCI remaining in the system is imposed by the active transmissions of the tierone BSs that belong to $\mathbf{B}_{o}$. Hence, the discrete-time model of the received signal at MS $j$ may be written as:

$$
\mathbf{y}_{j}=\mathbf{H}_{j} \mathbf{G}_{j} \mathbf{x}_{j}+\sum_{i=1, i \neq j}^{N_{u}} \mathbf{H}_{j} \mathbf{G}_{i} \mathbf{x}_{i}+\mathbf{\Psi}+\mathbf{n}_{j},
$$

where the first three terms of $\mathrm{Eq}$ (3) represent the desired signal of MS $j$, the Multi-User Interference (MUI) that is imposed by the simultaneous transmissions of by all BSs in the cooperative site and the $\mathrm{CCI}$ imposed by all the noncooperating tier-one BSs hosted in the set $\mathcal{B}_{o}$, respectively.

The difference in comparison to the system model of SCP discussed above is that $\mathbf{H}_{j} \in \mathbb{C}^{N_{r} \times N_{T}}$ denotes the joint MIMO channel matrix between the $N_{b}$ BSs in the cooperative site and the $j$ th MS, where we have $\mathbf{H}_{j}=$ $\left[\mathbf{H}_{1, j}, \mathbf{H}_{2, j}, \ldots, \mathbf{H}_{N_{b}, j}\right]$. Furthermore, $\mathbf{G}_{i} \in \mathbb{C}^{N_{T} \times N_{s}}, i \in$ $\left[1, N_{u}\right]$ denotes the joint linear precoding matrix of all BSs in the cooperative site intended for the $i$ th MS, where we have $\mathbf{G}_{i}=\left[\mathbf{G}_{1, i}^{T}, \mathbf{G}_{2, i}^{T}, \ldots, \mathbf{G}_{N_{b}, i}^{T}\right]^{T}$.

In MCP, the per-BS power constraint $P_{B S}$ should be satisfied, namely that [2] $\operatorname{Tr}\left[\sum_{i=1}^{N_{u}} \mathbf{G}_{k, i} \mathbf{G}_{k, i}^{H}\right]=P_{B S}, k \in \mathcal{B}_{c}$, where $\mathbf{G}_{k, i}$ represents the linear precoding matrix intended for the $i$ th MS employed at the $k$ th BS. In the case of MCP, the achievable rate is given as

$$
\mathcal{R}_{m}=\frac{1}{N_{u}} \sum_{j=1}^{N_{u}} \log \left|\mathbf{I}+\frac{\mathbf{H}_{j} \mathbf{G}_{j} \mathbf{G}_{j}^{H} \mathbf{H}_{j}^{H}}{\mathbf{R}_{I}+\sum_{i=1, i \neq j}^{N_{u}} \mathbf{H}_{j} \mathbf{G}_{i} \mathbf{G}_{i}^{H} \mathbf{H}_{j}^{H}}\right|,
$$

where Eq (4) is applicable to all linear precoding techniques considered in the next section except for the Joint TimeDivision (JTD) technique.

\section{B. Joint and Distributed Linear Precoding}

1) $J B F$ : The design principle of the linear Joint BeamForming (JBF) precoding matrix $\mathbf{G}_{i}$ is that all cooperative BSs jointly transmit in their respective directions maximising a specific MS's received signal power without aiming for minimising the MUI imposed, namely in the direction of all the $N_{s}$ right singular vectors corresponding to the largest $N_{s}$ singular values of the joint MIMO channel matrix $\mathbf{H}_{i}$.

2) JTD: The JTD technique follows the same design principle as JBF, with the slightly difference that only a single MS is served by all cooperative BSs at any instant in time. Hence, no MUI is imposed and the achievable rate is given by

$$
\mathcal{R}_{o}=\frac{1}{N_{u}} \sum_{j=1}^{N_{u}} \frac{1}{N_{u}} \log \left|\mathbf{I}+\mathbf{R}_{I}^{-1} \mathbf{H}_{j} \mathbf{G}_{j} \mathbf{G}_{j}^{H} \mathbf{H}_{j}^{H}\right|,
$$

which implies that the benefits of the logarithmic power gain achieved may be outweighed by the linear rate loss supporting only $1 / N_{u}$ of the total attainable rate.

3) JBD: The Joint Block Diagonalisation (JBD) technique proposed in [5] offers a different precoding method, which takes into account all the global MIMO channels denoted by 
$\mathbf{H}=\left[\mathbf{H}_{1}^{T}, \mathbf{H}_{2}^{T}, \ldots, \mathbf{H}_{N_{u}}^{T}\right]^{T}$. The JBD precoding matrix $\mathbf{G}_{j}$ configured for transmission to the $j$ th $\mathrm{MS}$ is constituted by two parts, namely $\mathbf{G}_{j}=\mathbf{G}_{j}^{0} \mathbf{G}_{j}^{1}$, where $\mathbf{G}_{j}^{0}$ is referred to as the nulling matrix, which lies in the null-space of all other joint MIMO channels $\mathbf{H}_{-j}=\left[\mathbf{H}_{1}^{T}, \ldots, \mathbf{H}_{j-1}^{T}, \mathbf{H}_{j+1}^{T}, \ldots, \mathbf{H}_{N_{u}}^{T}\right]^{T}$, hence we have $\mathbf{H}_{i} \mathbf{G}_{j}^{0}=\mathbf{0}, i \neq j$. On the other hand, the matrix $\mathbf{G}_{j}^{1}$, which was referred to as the maximising matrix, aims for maximising the received signal power of MS $j$ by transmitting in the directions of all the $N_{s}$ right singular vectors corresponding to the largest $N_{s}$ singular values of the effective channel $\mathbf{H}_{j} \mathbf{G}_{j}^{0}$. This nulling and maximising combination effectively eliminates the persistent MUI and at the same time obeys the JBF principle.

The precoding matrix generated above has to be obey the per-BS power constraint [2] $\operatorname{Tr}\left[\sum_{i=1}^{N_{u}} \mathbf{G}_{k, i} \mathbf{G}_{k, i}^{H}\right]=P_{B S}, k \in$ $\mathcal{B}_{c}$. Let $\mathbf{z}=\left[\mu_{1}, \ldots, \mu_{N_{b}}\right]^{T}$ denote the normalisation vector, whose entries are the normalisation factors corresponding to each of the cooperating BSs. Then the normalisation vector is given by solving the equation $\boldsymbol{\Omega} \mathbf{z}=P_{B S} \mathbf{1}_{N_{b}}$, where $\mathbf{1}_{N_{b}}$ represents the all-one column vector and $\boldsymbol{\Omega} \in \mathbb{R}^{N_{b} \times N_{b}}$ denotes the unconstrained power matrix, whose entry is the Frobenius norm of $\left\|\mathbf{G}_{k, i}\right\|_{F}^{2}$. When an illegitimate solution is found, the normalisation factor may be obtained as $\mu=$ $\min _{k \in \mathcal{B}_{c}} P_{B S}\left(\sum_{i=1}^{N_{u}}\left\|\mathbf{G}_{k, i}\right\|_{F}^{2}\right)^{-1}$ for all cooperating BSs.

4) JSLNR: The linear Joint Signal-to-Leakage-Noise-Ratio (JSLNR) precoding technique [6] aims for maximising the received signal power at the intended MS and at the same time for minimising the interference imposed on all other MSs. More explicitly, the SLNR $\gamma$ at MS $j$ is given by

$$
\gamma=\frac{\operatorname{Tr}\left[\mathbf{G}_{j}^{H} \mathbf{H}_{j}^{H} \mathbf{H}_{j} \mathbf{G}_{j}\right]}{\operatorname{Tr}\left[\mathbf{G}_{j}^{H}\left(\mathbf{\Theta} / P_{j}+\mathbf{H}_{-j}^{H} \mathbf{H}_{-j}\right) \mathbf{G}_{j}\right]},
$$

where the numerator denotes the signal power received at MS $j$, while the denominator represents the interference leakage power imposed on all other MSs by the transmission of MS $j$ and $\boldsymbol{\Theta}=\left\{N_{0} N_{r}+\sum_{i \in \mathcal{B}_{o}} \operatorname{Tr}\left[\mathbf{G}_{i, i}^{H} \mathbf{H}_{i, j}^{H} \mathbf{H}_{i, j} \mathbf{G}_{i, i}\right]\right\} \mathbf{I}_{N_{T}}$. Hence the optimisation problem may be stated as

$$
\mathbf{G}_{j}=\arg \max _{\mathbf{G}_{j}} \gamma .
$$

It can be seen that the maximisation problem of $\mathrm{Eq}$ (7) requires the knowledge of the power allocation $P_{j}=\sum_{k=1}^{N_{b}} P_{k, j}$, while satisfying the per-BS power constraint. In this paper, we adopt a simple proportional power allocation strategy of [7].

$$
P_{k, j}=\frac{\operatorname{Tr}\left[\mathbf{H}_{k, j} \mathbf{H}_{k, j}^{H}\right]}{\operatorname{Tr}\left[\sum_{i=1}^{N_{u}} \mathbf{H}_{k, i} \mathbf{H}_{k, i}^{H}\right]} P_{B S} .
$$

Furthermore, the optimisation problem of $\mathrm{Eq}$ (7) can be decoupled into the individual optimisations by forcing $\mathbf{G}_{j}$ to be an orthonormal matrix as discussed in [6], where we have $\mathbf{G}_{j}=\operatorname{eigv}\left(\mathbf{B}^{-1} \mathbf{A}\right)$, representing the eigenvectors corresponding to the $N_{s}$ largest eigenvalues of $\mathbf{B}^{-1} \mathbf{A}$, with $\mathbf{B}=\mathbf{\Theta} / P_{j}+\mathbf{H}_{-j}^{H} \mathbf{H}_{-j}$ and $\mathbf{A}=\mathbf{H}_{j}^{H} \mathbf{H}_{j}$. Finally, the resultant linear precoding matrix $\mathbf{G}_{k, j}$ is multiplied by the appropriately allocated power $P_{k, j} / N_{s}$.

5) DSLNR: Instead of maximising the SLNR $\gamma$ of Eq (6) for a given transmitted signal power from all cooperative BSs and leakage power generated by all cooperative BSs with the aid of the global CSI, alternatively the Distributed Signal-toLeakage-Noise-Ratio (DSLNR) maximises the SLNR $\gamma_{p}$ that computes the signal power transmitted from and the leakage power generated by individual BS only may be defined as [7]:

$$
\gamma_{p}=\frac{\operatorname{Tr}\left[\mathbf{G}_{k, j}^{H} \mathbf{H}_{k, j}^{H} \mathbf{H}_{k, j} \mathbf{G}_{k, j}^{H}\right]}{\operatorname{Tr}\left[\mathbf{G}_{k, j}^{H}\left(\boldsymbol{\Theta} / P_{k, j}+\sum_{i=1, i \neq j}^{N_{u}} \mathbf{H}_{k, i}^{H} \mathbf{H}_{k, i}\right) \mathbf{G}_{k, j}^{H}\right]},
$$

where $P_{k, j}$ is given by Eq (8) and the solution to this partial SLNR optimisation problem follows the same principle as the above-mentioned JSLNR linear precoding technique. The resultant achievable rate of DSLNR is also given by Eq (4). Note that as a result of the partial SLNR optimisation, each BS computes its precoding matrix for each MS locally, based on the local CSI $\mathbf{H}_{k, i}, i \in\left[1, N_{u}\right]$ for BS $k$. Hence, this arrangement operates on the basis of distributed principles.

\section{Centralised and Decentralised CSI-DT}

An inherent assumption, when designing the above linear precoding matrices is that the global CSI $\mathbf{H}=$ $\left[\mathbf{H}_{1}^{T}, \mathbf{H}_{2}^{T}, \ldots, \mathbf{H}_{N_{u}}^{T}\right]^{T}$ should be made available to all the cooperating BSs. There are two MCP frameworks, which may be differentiated according to how this global CSI is shared amongst the multiple BSs.

1) Centralised Framework: In the centralised framework, the $\mathrm{CSI}_{\mathbf{H}}$ of all cooperating BSs with respect to the $j$ th MS is estimated at the $j$ th MS's receiver and then it is fed back to its anchor BS in quantised form. Then, the quantised CSI $\hat{\mathbf{H}}_{j}$ received at each individual BS is reported to the $\mathrm{CU}$ in order to form the quantised global CSI $\hat{\mathbf{H}}$. Finally, the CU jointly performs linear precoding and in turn sends back the precoding matrices to each BS. Note that this framework is not applicable to the DSLNR linear precoding technique.

2) Decentralised Framework: On the other hand, in the decentralised framework [3], the $j$ th MS sends back its estimated and quantised CSI $\hat{\mathbf{H}}_{j}$ to all the cooperative BSs involved through individual feedback links. Each cooperative BS then unilaterally optimises the linear precoding matrix, based on its own version of the quantised global CSI $\hat{\mathbf{H}}_{j}, j \in\left[1, N_{u}\right]$ that was gathered from all MSs' feedback. Thus, the decentralised framework avoids the requirement of exchanging the CSI as well as the precoding matrix via the back-haul network, which would impose an extra computational load on each local BS.

3) Quantised CSI: To elaborate a little further, we assume that MS $j$ is capable of accurately estimating the joint MIMO channel $\mathbf{H}_{j}$ and employs the Random Vector Quantiser (RVQ) [8] for quantising the Channel Direction Information (CDI) of $\mathbf{H}_{j}$, while the norm of $\mathbf{H}_{j}$ representing the Channel Quality Information (CQI) is assumed to be perfectly known at the cooperative BSs. This implies that a CSI quantisation codebook $\mathbf{C}=\left[\mathbf{C}_{1}, \mathbf{C}_{2}, \ldots, \mathbf{C}_{N_{c}}\right]$ consisting $N_{c}=2^{b}$ zero-mean unit-norm complex Gaussian matrices $\mathbf{C}_{k} \in \mathbb{C}^{N_{r} \times N_{T}}, k \in$ $\left[1, N_{c}\right]$ is constructed and made available to both the MS's receiver and to the cooperative $\mathrm{BSs} / \mathrm{CU}$, where $b$ denotes the number of quantisation bits. In the quantised feedback regime, the specific $b$ bits representing the specific codebook index of $k=\max _{k \in\left[1, N_{c}\right]}\left|\cos \left[\angle\left(\mathbf{H}_{j}, \mathbf{C}_{k}\right)\right]\right|$ are transmitted, where the codebook may be designed to satisfy different design 
criteria. At the transmitter side, these received codebookindex bits, which may be prone to feedback errors, are used to regenerate the quantised CSI $\hat{\mathbf{H}}_{i}$ by combining them with the perfectly known CQI value and with the quantised CDI stored in codebook C. Moreover, each of the $b$ bits is assumed to experience independent feedback errors with an error probability of $P_{e}$. As discussed in [3], there are a total of $N_{b}$ feedback links involved in the centralised framework, while as many as $N_{b} N_{u}$ feedback links may be observed in the decentralised framework. Hence the latter is more sensitive to the detrimental effects of the quantised CSI, since differently quantised global CSI may be observed at different BSs.

\section{NUMERICAL RESULTS}

We now provide numerical results for charactering the various linear precoding techniques under both frameworks in two different scenarios, namely the fully-loaded scenario $\left(N_{T}=\right.$ $N_{R}$ ) of $\left\{N_{b}, N_{t}, N_{u}, N_{r}, N_{s}\right\}=\{3,2,3,2,2\}$ and the lightlyloaded scenario $\left(N_{T}>N_{R}\right)$ of $\left\{N_{b}, N_{t}, N_{u}, N_{r}, N_{s}\right\}=$ $\{3,4,3,2,2\}$.

We considered the so-called Urban Micro setup [9], where the BS-to-BS distance was defined as $D=1000 \mathrm{~m}$. The MIMO channel of each BS-MS pair is constituted by three components, i.e.by $\mathbf{H}_{i, j}=\left(A_{i, j}^{l} A_{i, j}^{s}\right)^{1 / 2} \mathbf{H}_{i, j}^{f}$. Explicitly, $\mathbf{H}_{i, j}^{f} \in \mathbb{C}^{N_{r} \times N_{t}}$ represents the fast fading component, which is assumed to be frequency-flat with zero-mean and unityvariance complex Gaussian entries, $A_{i, j}^{l}$ denotes the pathloss component and $A_{i, j}^{s}=10^{\xi / 10}$ is the lognormal shadow fading component, where $\xi$ is generated by a zero-mean real-valued Gaussian random variable having a standard derivation of $\sigma_{s}=8 \mathrm{~dB}$. The pathloss model is given by $A_{i, j}^{l}=\beta d_{i, j}^{\alpha}$, where $d$ denotes the BS-MS distance in meter and $[\alpha, \beta]=$ $\left[-3,1.35 \times 10^{7}\right][10]$. Furthermore, we let the Signal-to-Noise Ratio (SNR) at the cell-edge area be $25 \mathrm{~dB}$.

In each simulation, three MS locations are generated randomly and independently, where each of the MSs is uniformly positioned in the circle determined by the radius of $r=$ $0.1 D$ and angle range of $120^{\circ}$, corresponding to the sector covered by its anchor BS. Our numerical results are obtained by investigating 100 independent simulations, where each simulation was terminated after 5000 simulation runs. Due to space limit, we only present results for $b=10$ quantisation bits and for a feedback error probability of $P_{e}=0.01$ based on the argument that using $b=10$ bits for feedback information is technically meaningful.

\section{A. Fully-loaded Scenario}

Fig 2(a) and Fig 2(b) show the outage rate of the various linear precoding techniques considered, when using perfect CSI-DT (top subplot) and quantised CSI-DT (bottom subplot) in the fully-loaded scenario by assuming no tier-one CCI seen and full tier-one CCI, respectively. When perfect CSIDT is available, the performance of both the centralised and decentralised CSI-DT framework is identical, hence only the centralised framework is characterised where appropriate.

The results of the top subplot of Fig 2(a) suggest that the DSLNR precoding technique is the worst amongst the linear precoders considered. It also shows that the JBD has the best outage rate of all the linear precoding techniques considered. On the other hand, when quantised CSI-DT is considered, we only characterise the decentralised CSI-DT framework for the best linear precoding technique. We observe in the bottom subplot of Fig 2(a) that remarkably, the simple JTD technique marked by the dotted line performs best under the decentralised CSI-DT framework, while the other MCP linear precoding techniques suffer from a substantial rate loss compared to that of assuming perfect CSI-DT, as shown in the top subplot of Fig 2(a). The advantage of the JSLNR and the JBD techniques over the naive JBF and over the flexible DSLNR erodes in the presence of quantisation.

In the top subplot of Fig 2(b) assuming perfect CSI-DT, the linear JBD precoding technique represented by the dash-dot line becomes the worst technique and its outage rate is close to that of the SCP due to its poor performance at low SINRs. However, both the JTD and JBF techniques are more robust in this operating range, as indicated by the dotted line and dashed line, respectively. Moreover, the JSLNR achieves the highest outage rate. Remarkably, the DSLNR technique becomes the runner up, which was the worst choice in the top subplot of Fig 2(a). When quantised CSI-DT is considered, the DSLNR operating under the decentralised CSI-DT framework becomes noticeably superior to all other linear precoding techniques under the centralised CSI-DT framework, despite the fact that all linear MCP precoding techniques suffers from the CSI quantisation, which imposes an outage rate loss.

\section{B. Lightly-loaded Scenario}

Similar to Fig 2(a), Fig 2(c) shows the outage rate for a lightly-loaded scenario. The top subplot demonstrates that in contrast to the top subplot of Fig 2(a), the linear DSLNR precoding technique achieves a remarkable performance improvement in lightly-loaded scenario. This performance improvement of the linear DSLNR precoding technique compared to the fully-loaded scenario is achieved, because as a benefit of having a relatively high number of transmit antennas at each BS provides an extra DoF to combat the detrimental effects of the MUI. When quantised CSI-DT is used, as characterised in the bottom subplot of Fig 2(c), the advantage of most of the linear precoding techniques erodes and the JTD operating in the decentralised framework becomes the best. Finally, similar to Fig 2(b), Fig 2(d) portrays the outage rate for the lightly-loaded scenario. Although the JBD performed worst in the top subplot of Fig 2(b), as indicated by the dash-dot line, it achieves a higher outage rate than all other linear precoding techniques. When quantised CSI-DT is considered, the DSLNR precoding technique achieves a higher outage rate under the decentralised framework, than any of the other linear precoding techniques under the centralised framework.

\section{CONCLUSION}

In this paper, we characterised the outage rate of various linear precoding techniques in conjunction with two CSI exchange frameworks. We employed realistic CSI quantisation and feedback under both a fully-loaded scenario and an 

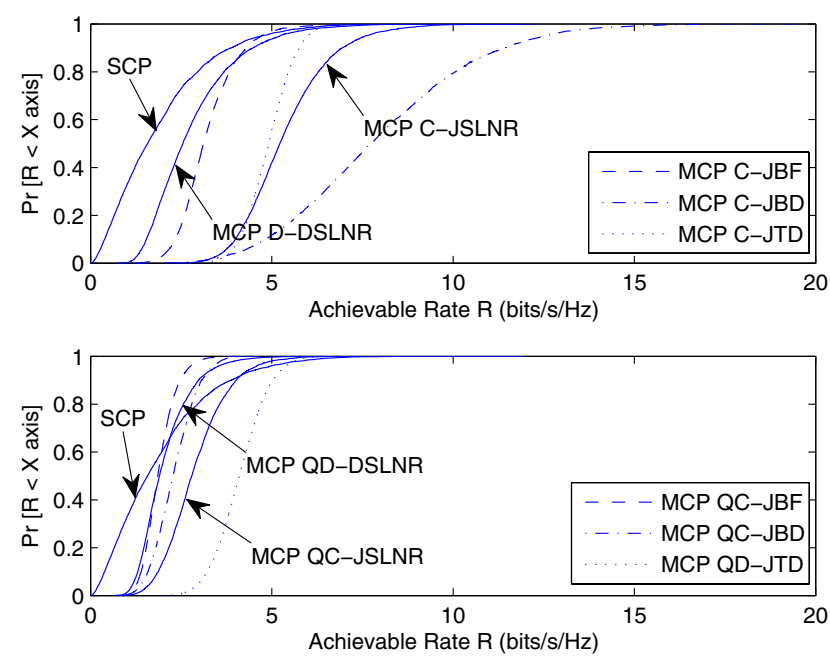

(a) fully-loaded scenario no tier-one CCI
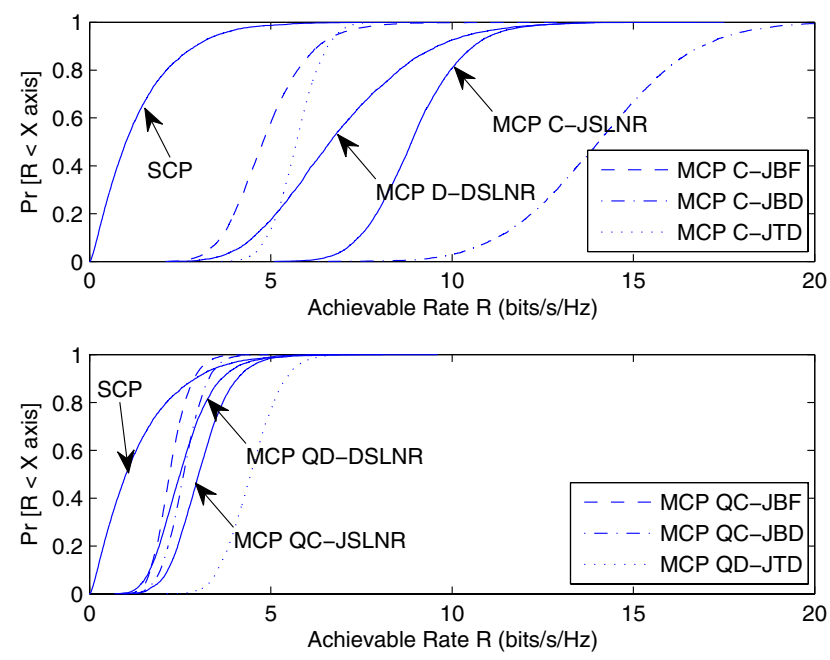

(c) lightly-loaded scenario no tier-one CCI
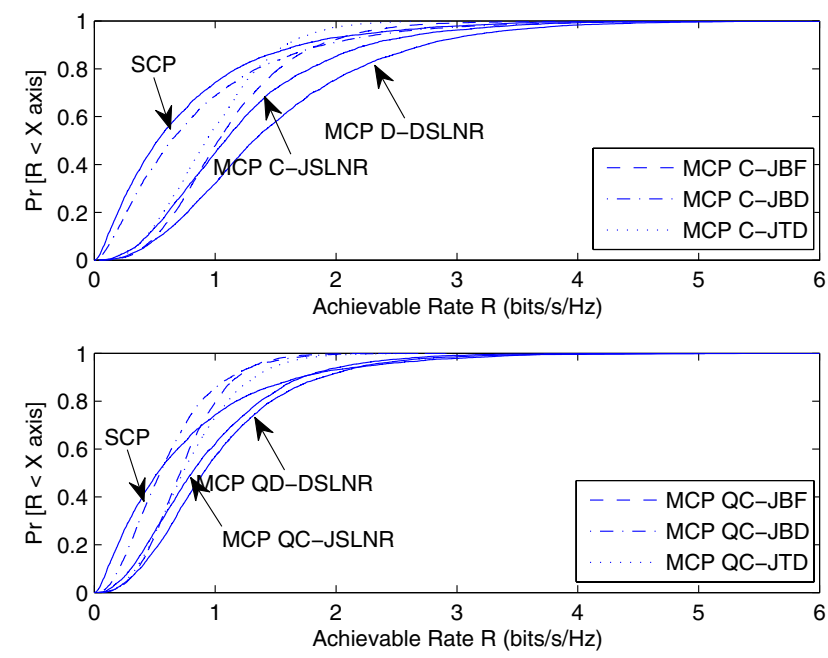

(b) fully-loaded scenario full tier-one CCI
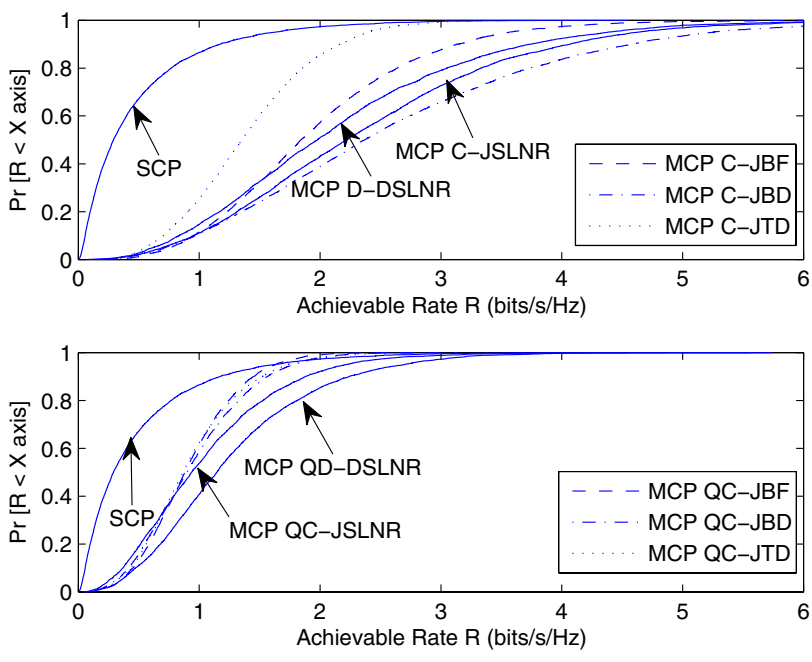

(d) lightly-loaded scenario full tier-one CCI

Fig. 2. Outage rate of various linear precoding techniques using perfect CSI-DT (top subplot) and quantised CSI-DT (bottom subplot). The terminology of 'QC / QD-linear precoding technique' represents the linear precoding technique operating in the quantised-centralised / quantised-decentralised framework.

lightly-loaded scenario. Numerical results demonstrated that the DSLNR operating in the decentralised framework when considering quantised CSI scenario has the best outage rate performance, when full tier-one CCI persists. It also has the simplest cooperative strategy, since only local precoding decisions have to be made. By contrast, the low-complexity JTD operating in the decentralised framework exhibits superiority in the quantised CSI scenario, when no tier-one CCI exists, where it dispenses with sophisticated precoding techniques that require complex matrix manipulation.

\section{REFERENCES}

[1] M. Karakayali, G. Foschini, and R. Valenzuela, "Network coordination for spectrally efficient communications in cellular systems," IEEE Wireless Communications Magazine, vol. 13, pp. 56-61, Aug 2006.

[2] H. Zhang and H. Dai, "Cochannel interference mitigation and cooperative processing in downlink multicell multiuser MIMO networks," EURASIP Journal on Wireless Communications and Networking, vol. 2004, pp. 222-235, Dec 2004
[3] A. Papadogiannis, E. Hardouin, and D. Gesbert, "Decentralising multicell cooperative processing: a novel robust framework," EURASIP Journal on Wireless Communications and Networking, vol. 2009, pp. 222-235, Apr 2009.

[4] D. Love, R. Heath, V. Lau, D. Gesbert, B. Rao, and M. Andrews, "An overview of limited feedback in wireless communication systems," IEEE Journal on Selected Areas in Communications, vol. 26, pp. 1341-1365, Oct 2008.

[5] Q. Spencer, A. Swindlehurst, and M. Haardt, "Zero-forcing methods for downlink spatial multiplexing in multiuser MIMO channels," IEEE Transactions on Signal Processing, vol. 52, pp. 461-471, Feb 2004.

[6] M. Sadek, A. Tarighat, and A. Sayed, "A leakage-based precoding scheme for downlink multi-user MIMO channels," IEEE Transactions on Wireless Communications, vol. 6, pp. 1711-1721, May 2007.

[7] E. Bjornson, R. Zakhour, D. Gesbert, and B. Ottersten, "Cooperative multicell precoding: Rate region characterization and distributed strategies with instantaneous and statistical CSI," submitted to IEEE Transactions on Signal Processing.

[8] N. Jindal, "MIMO broadcast channels with finite rate feedback," IEEE Transactions on Information Theory, vol. 52, pp. 5045-5059, June 2006.

[9] R. Steele and L. Hanzo, Mobile Radio Communications. New York, USA: IEEE Press - John Wiley, 1999.

[10] T. S. Rappaport, Wireless Communications: Principles and Practice. Englewood Cliffs, NJ, USA: Prentice-Hall, 1996. 